\title{
New PWM method and commutation strategy for HF-link converters for fuel cells and photovoltaics
}

Ljusev, Petar; Andersen, Michael Andreas E.

Published in:

IEEE Industry Applications Society

Link to article, DOI:

10.1109/IAS.2005.1518667

Publication date:

2005

Document Version

Publisher's PDF, also known as Version of record

Link back to DTU Orbit

Citation (APA):

Ljusev, P., \& Andersen, M. A. E. (2005). New PWM method and commutation strategy for HF-link converters for fuel cells and photovoltaics. In IEEE Industry Applications Society (Vol. 3). IEEE.

https://doi.org/10.1109/IAS.2005.1518667

\section{General rights}

Copyright and moral rights for the publications made accessible in the public portal are retained by the authors and/or other copyright owners and it is a condition of accessing publications that users recognise and abide by the legal requirements associated with these rights.

- Users may download and print one copy of any publication from the public portal for the purpose of private study or research.

- You may not further distribute the material or use it for any profit-making activity or commercial gain

- You may freely distribute the URL identifying the publication in the public portal 


\title{
New PWM method and commutation strategy for HF-link converters for fuel cells and photovoltaics
}

\author{
Petar Ljušev and Michael A.E. Andersen \\ Ørsted • DTU Automation, Technical University of Denmark \\ Elektrovej, DTU Building 325, DK-2800 Kgs. Lyngby, Denmark \\ E-mail: pl@oersted.dtu.dk, ma@oersted.dtu.dk
}

\begin{abstract}
This paper presents a new PWM method and commutation strategy for HF-link converters, which leads to safe commutation of the load current in the output bidirectional bridge. The proposed implementation is independent of the particular HF-link converter topology and bidirectional switch selection and is therefore usable at all output power levels and switching frequencies. Theoretical analysis of the approach and experimental investigation are presented to prove the feasibility of the concept.
\end{abstract}

\section{INTRODUCTION}

HF-link converters [1] have been proposed as a compact and highly efficient solution in a wide range of applications: from direct single-phase and multi-phase DC-AC converters [2], [3], direct AC-AC converters [4], Uninterruptible Power Supplies (UPSs) [5], [6], three-phase electrical drives for connection to single-phase utility grid [7], converters for renewables Fuel Cells (FCs) [8] and Photovoltaics (PVs), to audio power amplifiers [9], [10], [11]. As their name suggests, HF-link converters replace the common DC-link found in conventional voltage-source and current-source inverters with a HF-link, avoiding the use of bulky reactive components for filtering the DC-link. As a result the secondary side diode rectifiers associated with the DC-link are removed, the energy flow is kept bidirectional over the isolation barrier and the number of energy conversions is reduced, increasing the converter efficiency. The latter advantage is rather important for FCs and PVs, which have low conversion efficiency (maximum of around $60 \%$ efficiency for certain types of FCs with combined electrical and thermal cycle, with pure hydrogen as fuel) and low output voltages, so the electrical power converters must be very efficient in order not to spoil overall system efficiency.

HF-link converters represent very interesting solution for the cost-driven segment of market, since they minimize the use of bulky reactive components like inductors and electrolytic capacitors, whose development is rather slow due to the limitations in the available magnetic and dielectric materials and on the other side promote use of semiconductor components, which improve their properties and become cheaper on almost a daily basis. Thus, HF-link converters can be compared with the "all-silicon" three-phase matrix converters as another direct energy conversion approach, but without isolation. Although HF-link conversion has been known for several decades, its application has been limited due to practical implementation problems, primarily with the safe commutation of the load current in the secondary-side bidirectional bridge.

The introduction of the HF-link in a form of isolation transformer has tremendous implications on this class of converters. The most important one is probably the need for constructing a switching output stage with bidirectional switches, where the load current freewheeling path is non-existent and therefore the load current commutation between the outgoing and incoming switches depends heavily on implementing smart commutation techniques. Simple switch control techniques like overlapping conduction and dead-time insertion result in shoot-through and overvoltage phenomena respectively, that are adversely effecting both the reliability and efficiency of the power converters. Most of the advanced commutation techniques from the threephase matrix converters are not applicable to the single-phase HF-link converters, and the utilization of expensive current and voltage sensors for detecting their polarities is for most of the cases economically not feasible in the lower power range. Nonetheless, safe commutation of the secondary-side bidirectional bridge is still possible by utilizing the fact that in almost all the topologies, the HF-link voltage has a rectangular shape and is therefore easy to detect its polarity out of the transition instants. This information can be used to decide about the proper switching sequence leading to smooth current commutation [12], without using any additional circuitry or semiconductor components with self-turn-off characteristics.

This paper starts with a brief overview of the possible HFlink topologies and their time and frequency domain analysis. Subsequently a new PWM method and commutation strategy for HF-link converters that leads to safe commutation of the load current will be presented. These will be implemented on a HF-link converter with non-modulated transformer voltages i.e. converters where the HF-link voltage represents rectangular waveform with duty cycle of $50 \%$ which is processed further by the output stage with bidirectional switches. Finally, experimental results of a prototype will be presented.

\section{HF-LINK CONVERSION}

\section{A. Converter with LF transformer}

The inverter with Low Frequency (LF) transformer, shown in Fig. 1, is characterized with its simple structure, consisting of a single switching stage on the primary side. LF transformer output voltage is directly filtered on the secondary side using an LC filter to deliver the desired load voltage waveform. 


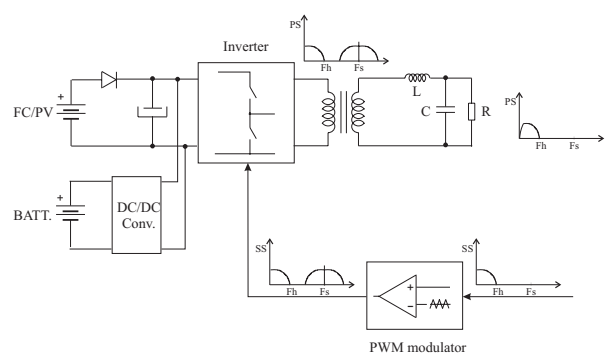

Fig. 1. Converter with LF transformer

The reference voltage waveform with its Signal Spectrum (SS) limited to the highest frequency $F_{h}$ enters the PWM modulator. The SS of the PWM modulator output depends on the carrier shape (ex. single-sided or double-sided triangular carrier) and the number of levels voltage levels (ex. 2-level or 3-level), and is including both the reference voltage waveform baseband, carrier harmonics as well as their intermodulation product sidebands. When the output of the PWM modulator is directly driving the input stage, the Power Spectrum (PS) of the transformer voltage has a LF content that can extend down to $50 / 60 \mathrm{~Hz}$ for utility grid or $400 \mathrm{~Hz}$ for special aircraft power systems, demanding huge isolation transformer. Therefore this approach is regarded as economically unsound.

\section{B. HF-link converter with modulated voltages}

HF-converter with modulated transformer voltages [2], shown in Fig. 2, uses HF 3-level PWM signal to drive the primary switching stage and the HF transformer, where the LF content from the PWM modulator output has been eliminated by multiplying it with a rectangular signal synchronized with the triangular carrier. On the secondary side, the bidirectional bridge is driven with rectangular signal to restore the reference voltage waveform baseband. The main advantage of this approach is that the transformer voltage contains periods with zero voltage each switching period, which helps the commutation of the secondary side bidirectional bridge and lowers its switching losses. The aforementioned benefit is achieved by shortly turning on both the outgoing and incoming bidirectional switches during the periods with zero transformer voltage, which does not create short-circuit current on the primary side and in the same time provides an uninterrupted path for the load current. In the same time, due to the 3-level phase-shifted PWM modulation on the primary side, the flux density excursion in the transformer is reduced at low output voltage levels. This approach has some disadvantages though, like the need for high switching frequencies in both switching stages which increases the losses, 3-level PWM topologies, fairly high level of switching synchronization needed, as well the inability to build auxiliary power supplies using the same power transformer, since the power flow is dependant on the output voltage level.

\section{HF-link converter with non-modulated voltages}

HF-link converter with non-modulated transformer voltages, shown in Fig. 3, is driving the primary-side stage and the

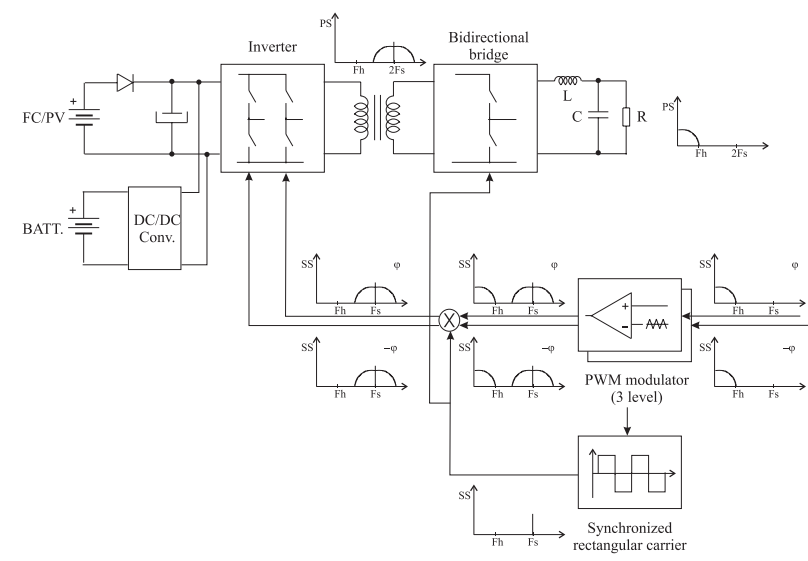

Fig. 2. HF-link converter with modulated transformer voltages

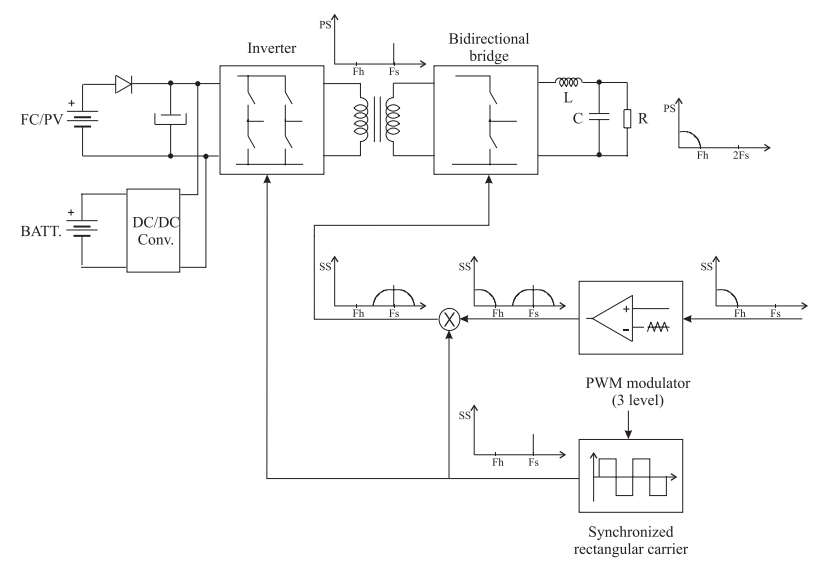

Fig. 3. HF-link converter with non-modulated transformer voltages

transformer with the 50\% duty cycle HF rectangular waveform. The rest of the coding and decoding of the LF reference voltage waveform is done on the secondary side, where the bidirectional bridge is driven with the PWM signal being a product of the output of the PWM modulator and the same rectangular carrier. The main benefits of this approach are the simplicity of the primary-side control, reduced input current differential-mode EMI, easy derivation of auxiliary voltages for control biasing or isolated gate drive supplies by fitting additional windings on the same core, as well as the possibility of implementing different switching frequencies on the primary and secondary side which allows for loss optimization with regard to the given thermal interface. However, the commutation of the secondary side bidirectional bridge becomes rather involved and depends heavily on using load voltage clamps [11], [5] or advanced commutation techniques [12]. Due to the numerous advantages of the HF-link converters with non-modulated voltages, this approach has been chosen for implementation and is discussed in detail in the next sections.

Many HF-link converter solutions proposed for high power applications have been implemented using thyristors, where the self turn-off property of the latter and the implemented 


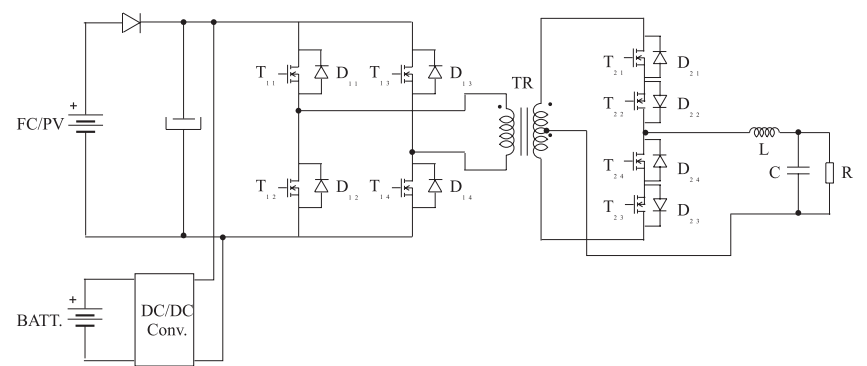

Fig. 4. HF-link converter with MOSFETs for FC/PV system

natural commutation simplify the load current commutation. Unfortunately, limited switching speed of the self turn-off power components does not allow for significant reduction of output filter size. Therefore, the objective of this work was to develop a modulation method, combined with a safecommutation strategy, that will allow use of faster power components (MOSFETs, IGBTs etc.) in HF-link converters, like the one presented in Fig. 4.

\section{NEW PWM METHOD FOR HF-CONVERTERS WITH NON-MODULATED TRANSFORMER VOLTAGES}

In this section, the new PWM method will be derived and its frequency spectrum will be determined for the case where the PWM modulator triangular carrier frequency $f_{c 2}$, equal to the output stage switching frequency $f_{s 2}$ is two times the input stage switching frequency $f_{H F}$, i.e. $f_{c 2}=2 f_{H F}$. At the end, the frequency spectrum of the new PWM method will be derived for the general case $f_{c 2}=r f_{H F}$, where the ratio $r$ is even integer number.

The reason for having lower HF-link frequency $f_{H F}$ i.e. lower input stage switching frequency $f_{s 1}$ compared to the output stage switching frequency $f_{s 2}$ hides in the particular application of these HF-link converters for interfacing predominantly low voltage fuel cells and photovoltaics to usually higher voltage DC or AC power systems. Power switches in the primary-side input stage conduct much higher currents directly from these low voltage sources, which limits their switching speed due to the unavoidable parasitics. On the other hand, increasing the switching frequency of the power switches in the secondary-side output stage has beneficial effects on decreasing the size of downstream output filter, making it much smaller.

\section{A. Derivation of the new PWM method}

Taking a closer look at the PWM modulator section in Fig. 3 it becomes apparent that the switching frequency of the secondary-side bidirectional bridge $f_{s 2}$ is a sum of PWM modulator carrier frequency $f_{c 2}$ and the frequency of the HFlink $f_{H F}, f_{s 2}=f_{H F}+f_{c 2}$. This is a result of the secondaryside gate drive being a product of the PWM modulator output and the rectangular waveform driving the primary-side inverter stage, so that the bidirectional bridge is switching at each HFlink voltage polarity change to provide output voltage that is exactly the same as the PWM modulator output. This is shown
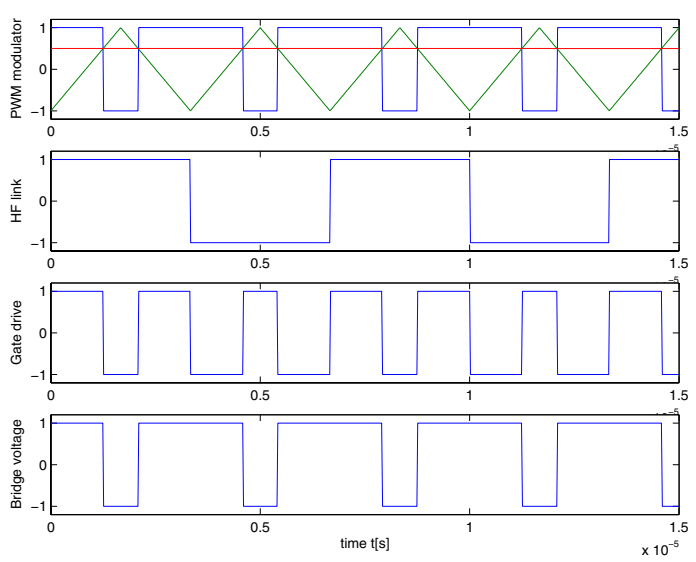

Fig. 5. PWM method with 3 switchings during one period $T_{c 2}$

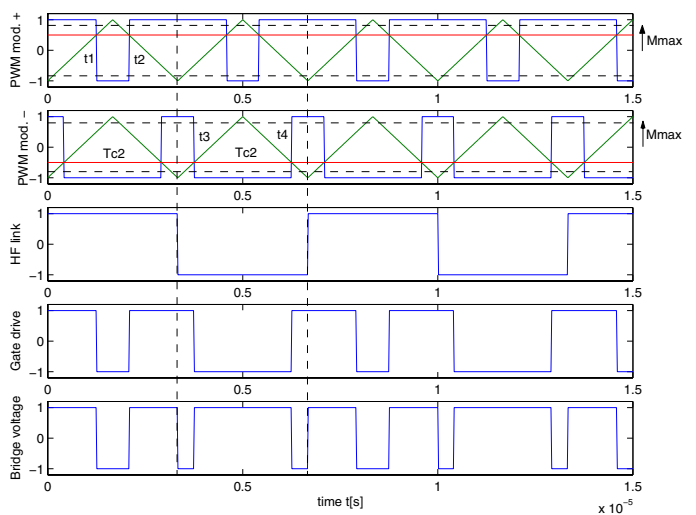

Fig. 6. Proposed PWM method with 2 switchings during one period $T_{c 2}$

in Fig. 5, where the gate drive waveform changes three times per period of the triangular carrier.

The switching frequency of the bidirectional bridge $f_{s 2}$ can be made equal to the carrier frequency $f_{c 2}$ and therefore result in two switchings per carrier period $T_{c 2}$ by giving up the desire to exactly restore the PWM modulator output across the output filter and instead choosing a switching waveform that will give the same average value of the bridge output voltage, like the original PWM modulator waveform. This means that the switching of the bidirectional bridge is not performed as soon as the HF-link changes polarity, but the switching time instants are readjusted. Looking at Fig. 6, this means that the average values of the bridge output voltage in the first two carrier periods are the same, despite the fact that the exact waveform is different. Time instants $t_{1}$ and $t_{2}$ defining the edges of the negative output voltage during the half-period with positive HF-link voltage are given by:

$$
\begin{aligned}
t_{1} & =\frac{T_{c 2}}{4}\left(1+\frac{2 v_{\text {ref }}}{V_{\text {cmax }}}\right) \\
t_{2} & =\frac{T_{c 2}}{4}\left(3-\frac{2 v_{\text {ref }}}{V_{\text {cmax }}}\right)
\end{aligned}
$$

where $v_{r e f}$ is the reference voltage and $V_{c \max }$ is the maximum carrier voltage. From (1), the time interval $\Delta t_{-}$during which 

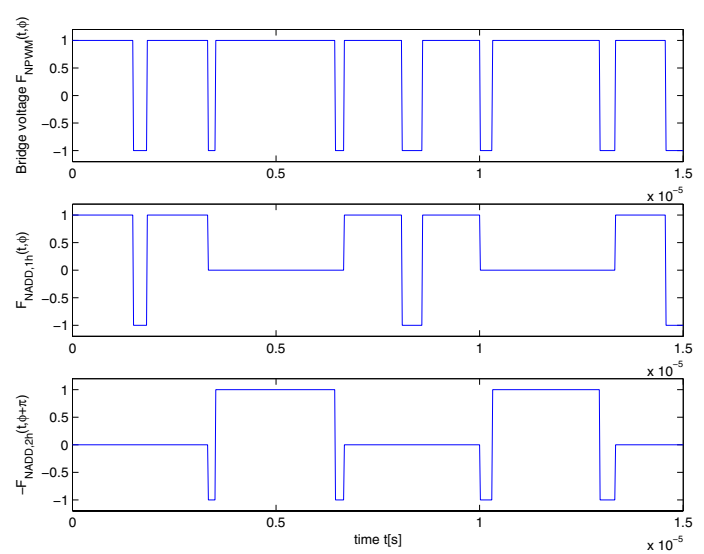

Fig. 7. The new PWM pulse train (top) and its two constitutive parts (below)

negative voltage is applied across the output filter is:

$$
\Delta t_{-}=t_{2}-t_{1}=\frac{T_{c 2}}{2}\left(1-\frac{2 v_{r e f}}{V_{c \max }}\right)
$$

During the second period $T_{c 2}$ when the HF-link voltage is negative, negative output voltage is applied at both ends of the carrier period and in order to have the same average output voltage like in the first period $T_{c 2}$, the time instants $t_{3}$ and $t_{4}$ defining the edges of the negative output voltage must be equal to:

$$
\begin{aligned}
& t_{3}=\frac{\Delta t_{-}}{2}=\frac{T_{c 2}}{4}\left(1+\frac{-2 v_{r e f}}{V_{c \max }}\right) \\
& t_{4}=T_{c 2}-\frac{\Delta t_{-}}{2}=\frac{T_{c 2}}{4}\left(3-\frac{-2 v_{r e f}}{V_{c \max }}\right)
\end{aligned}
$$

From equations (3) it becomes apparent that the average voltage during either polarity of the HF-link voltage in Fig. 6 will be equal, if the reference voltage is used in the PWM modulator during positive HF-link voltages and negative reference voltage is used in the PWM modulator during negative HF-link voltages, with the same form of the carrier. As long as the frequency of the reference voltage waveform is much lower than the frequency of the modulator carrier, there will be no output voltage distortion originating from this signal manipulation.

B. Frequency spectrum of the new PWM method in the case of $f_{c 2}=2 f_{H F}$

It is rather interesting to compare the frequency spectrum of the bridge voltage of the conventional PWM method shown in Fig. 5, which is equal to frequency spectrum of the twolevel double-sided PWM signal $F_{N A D D}$, with the frequency spectrum of the bridge voltage in the case of the proposed PWM method in Fig. 6. The analysis in this section will focus on the case where the carrier frequency in the PWM modulator $f_{c 2}$ is two times the HF-link switching frequency $f_{H F}$, while the general case will be presented in the following section.

The frequency spectrum of the proposed PWM method in Fig. 6 can be found by breaking the PWM pulse train into two separate groups of pulses that correspond to the periods with positive and negative HF-link voltage, correspondingly, as shown in Fig. 7. These separate PWM trains are result of the same PWM process $F_{N A D D, 1}$ with every second pulse skipped, where for the first pulse train the actual reference voltage and for the second time-shifted pulse train the negative reference voltage with subsequent reversal of the PWM pulses polarity due to the negative HF-link voltage is being used. At the end, the frequency spectra of the two PWM pulse trains are summed together to give the final frequency spectrum of the newly proposed PWM method $F_{N P W M}$.

The double Fourier series of a two-level, double-sided PWM with every second pulse skipped and pulses only in the beginning:

$$
\begin{aligned}
& F_{N A D D, 1}(t, \varphi)= \\
& 4 k M \cos \left(\omega_{m} t+\varphi\right)+ \\
& +2 \sum_{m=1}^{\infty}\left[\frac{2 \cos \left(2 \pi m k-\frac{\pi}{2}\right)}{m \pi} J_{0}(2 \pi m k M)+\right. \\
& \left.+\frac{\cos \left(4 \pi m k+\frac{\pi}{2}\right)}{m \pi}\right] \cdot \cos \left(m \omega_{H F} t\right)+ \\
& +4 \sum_{m=1}^{\infty} \sum_{n= \pm 1}^{\infty} \frac{\cos \left(2 \pi m k+\frac{(n-1) \pi}{2}-n \varphi\right)}{m \pi} . \\
& \cdot J_{n}(2 \pi m k M) \cos \left(m \omega_{H F} t+n \omega_{m} t+n \varphi\right)
\end{aligned}
$$

where $\omega_{m}$ and $\varphi$ are the angular frequency and the phase of the modulating signal, $\omega_{H F}$ is the angular frequency of the HF-link and $k=T_{c 2} /\left(4 \cdot T_{H F}\right)$ is a ratio of the carrier and input stage switching period, equal to $k=0.125$ for the case with two times higher carrier frequency $f_{c 2}$ then the HF-link frequency $f_{H F}$.

The double Fourier series $F_{N A D D, 1}$ given in equation (4) can be used as constitutive part to calculate the double Fourier series of the conventional two-level double-sided PWM bridge voltage shown at the bottom diagram in Fig. 5, as derived below:

$$
\begin{aligned}
& F_{N A D D}(t, \varphi)= \\
& =F_{N A D D, 1}(t, \varphi)+F_{N A D D, 1}\left(t-0.5 \frac{2 \pi}{\omega_{H F}}, \varphi\right)= \\
& 8 k M \cos \left(\omega_{m} t+\varphi\right)- \\
& -2 \sum_{m=1}^{\infty}\left[\frac{4 \sin \left(4 \pi m k-\frac{\pi}{2}\right) \sin (2 \pi m k)}{m \pi} J_{0}(2 \pi m k M)+\right. \\
& \left.+\frac{\cos \left(8 \pi m k+\frac{\pi}{2}\right)}{m \pi}\right] \cdot \cos \left(m \omega_{H F} t\right)- \\
& -8 \sum_{m=1}^{\infty} \sum_{n= \pm 1}^{\infty} \frac{\sin \left(4 \pi m k+\frac{(n-1) \pi}{2}-n\left(\varphi+\frac{\pi}{2}\right)\right)}{m \pi} . \\
& \quad \sin \left(2 \pi m k+\frac{n \pi}{2}\right) J_{n}(2 \pi m k M) . \\
& \quad \cdot \cos \left(m \omega_{H F} t+n \omega_{m} t+n \varphi\right)
\end{aligned}
$$




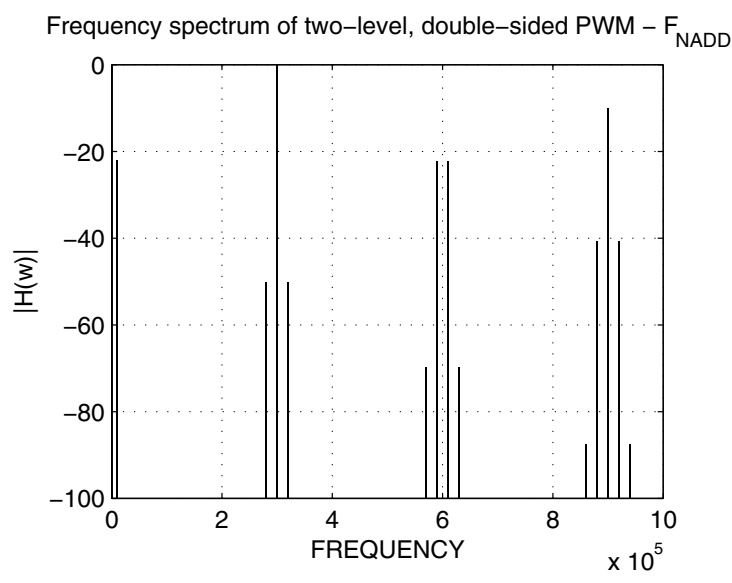

Fig. 8. Frequency spectrum of the conventional PWM method $F_{N A D D}$ with $M=0.1, f_{m}=10 \mathrm{kHz}, f_{H F}=150 \mathrm{kHz}, f_{c 2}=300 \mathrm{kHz}$

The magnitude diagram of the frequency spectrum of the conventional PWM bridge voltage $F_{N A D D}$ with modulation index equal to $M=0.1$, modulation frequency $f_{m}=10 \mathrm{kHz}$, HF-link frequency $f_{H F}=150 \mathrm{kHz}$ and triangular carrier frequency $f_{c 2}=300 \mathrm{kHz}$ is shown in Fig. 8 .

For calculating the double Fourier series of the newly proposed PWM method for the SICAM bridge voltage $F_{N P W M}$ shown at the bottom diagram in Fig. 6, previously developed equation (4)is used in the following way:

$$
\begin{aligned}
& F_{N P W M}(t, \varphi)= \\
& =F_{N A D D, 1}(t, \varphi)-F_{N A D D, 2}\left(t-0.5 \frac{2 \pi}{\omega_{H F}}, \varphi+\pi\right)= \\
& 8 k M \cos \left(\omega_{m} t+\varphi\right)+ \\
& +2 \sum_{m=1}^{\infty}\left[\frac{4 \cos \left(4 \pi m k-\frac{\pi}{2}\right) \cos (2 \pi m k)}{m \pi} J_{0}(2 \pi m k M)+\right. \\
& \left.\quad+\frac{2 \cos \left(4 \pi m k+\frac{\pi}{2}\right)+\cos \left(8 \pi m k+\frac{\pi}{2}\right)}{m \pi}\right] . \\
& \quad \cdot \cos \left(m \omega_{H F} t\right)+ \\
& +8 \sum_{m=1}^{\infty} \sum_{n= \pm 1}^{\infty} \frac{\cos \left(4 \pi m k+\frac{(n-1) \pi}{2}-n \varphi\right)}{m \pi} . \\
& \quad \cdot \cos (2 \pi m k) J_{n}(2 \pi m k M) \cos \left(m \omega_{H F} t+n \omega_{m} t+n \varphi\right)
\end{aligned}
$$

The magnitude diagram of the frequency spectrum of the new PWM bridge voltage $F_{N P W M}$ with modulation index of $M=0.1, f_{m}=10 \mathrm{kHz}, f_{H F}=150 \mathrm{kHz}$ and $f_{c 2}=300 \mathrm{kHz}$ is shown in Fig. 9. Amplitudes of the first five odd-order switching harmonics with varying modulation index $\mathrm{M}$ are given in Fig. 10.

It becomes apparent from Fig. 8 that the frequency spectrum of the conventional PWM method $F_{N A D D}$ in Fig. 5 comprises only of the modulating signal baseband, switching harmonics at multiples of the carrier frequency and their sidebands as
Frequency spectrum of the new two-level, double-sided PWM $-\mathrm{F}_{\text {NPWM }}$

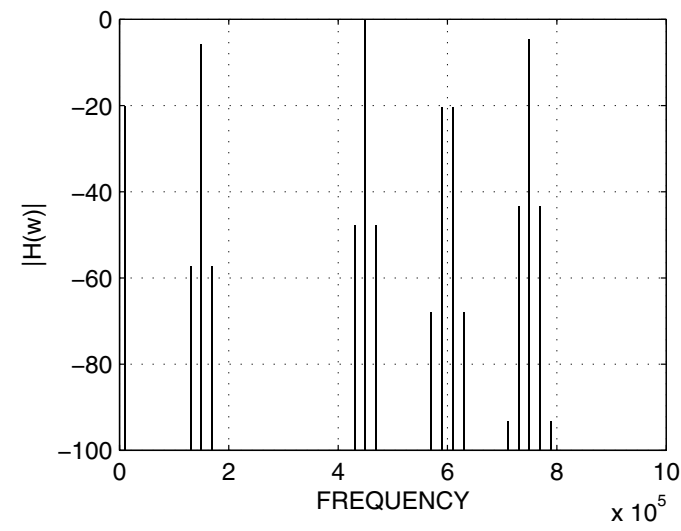

Fig. 9. Frequency spectrum of the new PWM method $F_{N P W M}$ with $M=0.1, f_{m}=10 \mathrm{kHz}, f_{H F}=150 \mathrm{kHz}, f_{c 2}=300 \mathrm{kHz}$

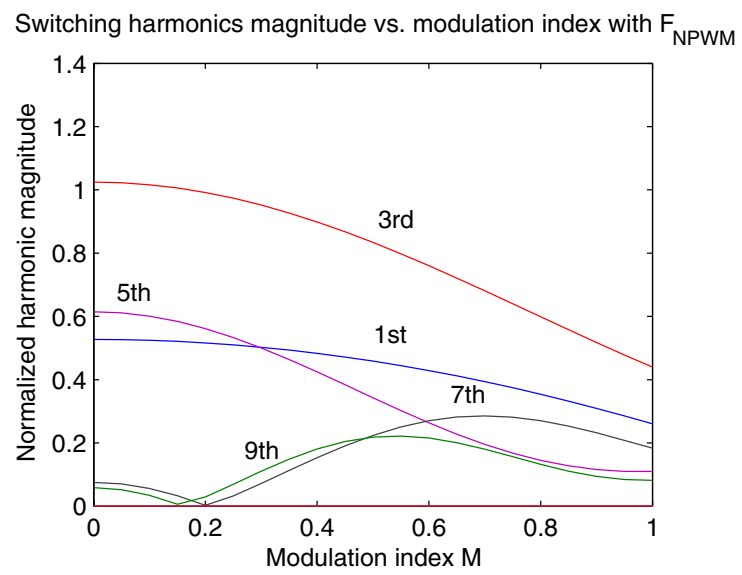

Fig. 10. Amplitude of the switching harmonics with varying modulation index

intermodulation product of the modulation baseband with the switching harmonics. As long as the PWM modulator triangular carrier frequency is substantially higher than the maximum modulating frequency $\omega_{c 2} \gg \omega_{m, \max }$, there are no switching frequency sidebands interfering with the audio baseband, and the modulation process is essentially linear. The switching harmonics amplitudes at the loudspeaker are suppressed by the output low-pass filter attenuation at those specific frequencies.

On the other hand, by closely inspecting Fig. 9 one can notice that the frequency spectrum of the proposed PWM for SICAMs $F_{N P W M}$ has intermodulation sidebands at the multiples of the input stage switching frequency i.e. HFlink frequency, which are not present in the conventional PWM $F_{N A D D}$, while some of the harmonics and sidebands associated with the PWM modulator carrier frequency have disappeared. Since the frequency of the HF-link is lower than the carrier frequency, these components are likely to cause larger ripple voltage across the loudspeaker, since the low- 
pass output filter is less effective at the HF-link frequency. Fortunately, the magnitude of the first harmonic of the HFlink voltage at the loudspeaker terminal is always lower than the third harmonic, which the output filter can attenuate more effectively.

\section{Frequency spectrum of the new PWM in general case}

The double Fourier series (4) of the two-level double-sided PWM signal in the beginning of the switching interval can be used to develop the double Fourier series of the new PWM with the desired even integer ratio $r$ of the triangular carrier $f_{c 2}$ and the HF-link frequency $f_{H F}, f_{c 2}=r f_{H F}$ :

$$
\begin{aligned}
& F_{N P W M}(t, \varphi)= \\
& \quad \sum_{i=1}^{r / 2} F_{N A D D, 1}\left[t-(i-1) \frac{2 \pi}{r \omega_{H F}}, \varphi\right]- \\
& \quad-\sum_{i=r / 2+1}^{r} F_{N A D D, 1}\left[t-(i-1) \frac{2 \pi}{r \omega_{H F}},-\varphi\right]
\end{aligned}
$$

with the coefficient $k=T_{c 2} /\left(4 \cdot T_{H F}\right)$.

\section{SAFE COMMUTATION OF THE BIDIRECTIONAL OUTPUT STAGE}

The proposed safe-commutation principle is based on the voltage-controlled commutation method known from the matrix converters, which utilizes the polarity of the HF-link voltage to choose the appropriate switching pattern when moving from the outgoing to the incoming bidirectional switch. Although the latter approach has been already used in conjunction with HF-link converters, its application together with the proposed PWM method can lead to significant simplification of the control circuitry. This is especially important when compared to the conventional PWM method shown in Fig. 5, where the switching of the output bidirectional stage must closely follow each switching of the input stage, which can be troublesome for the secondary-side voltage-controlled switching sequence if the transient process on the HF-link voltage has not finished before starting the aforementioned switching sequence.

The main challenge of the voltage-controlled commutation when used with the HF-link converters is keeping the same polarity of the HF-link voltage during the switching transition of the secondary-side bidirectional bridge. The proposed commutation method achieves this by limiting the modulation index of the reference voltage to $M_{\max }$ when entering the PWM modulator, which prohibits switching of the bidirectional bridge in the vicinity of the carrier peaks, like shown in Fig. 6. On the other hand, the switching of the primary-side inverter is synchronized with the peaks of the carrier, so there is no possibility of instantaneous switching of both stages. The safe-commutation switching pattern for the bidirectional bridge of the HF-link inverter in Fig. 4 is given in Tab. I, together with the markings of the natural commutation (o) and forced commutation $(\bullet)$. It should be emphasized that the correct switching sequence depends solely on the polarity of the HF-link voltage $v_{H F}$, while the direction of the output current $i_{o}$ determines only if the commutation is forced or natural. Stepping through the switching sequence is performed with a fixed commutation delay $\Delta t_{d}$, which is chosen depending on the switching speed of the used components and the allowed levels of output voltage distortion and switching losses. The duration of the natural commutation interval $\Delta t_{n c}$, during which the bridge voltage equals zero, depends on the transformer secondary-side voltage $V_{s}$, secondary leakage inductance $L_{s l}$ as well as the instantaneous value of the output current $i_{o}$ commutating between the switches:

$$
\Delta t_{n c}=2 L_{s l} \frac{\left|i_{o}\right|}{2 V_{s}}=\frac{L_{s l}\left|i_{o}\right|}{V_{s}}
$$

Switching losses in the output stage occur always with forced commutation, while during natural commutation switching losses are very low due to the low slope of the switch current, limited by the transformer leakage inductances. However, if the delay time between the switching steps in the sequence is not long enough to let the current commutate to the incoming switch naturally i.e. $\Delta t_{d}<\Delta t_{n c}$, some switching losses will occur due to the prematurely terminated natural commutation.

TABLE I

VOLTAGE CONTROLLED COMMUTATION SEQUENCE - NATURAL COMMUTATION (०) AND FORCED COMMUTATION (•)

\begin{tabular}{|c|c|c||c|c|c|c||c|}
\hline outgoing & $v_{H F}$ & $i_{O}$ & on & off & on & off & incoming \\
\hline \hline \multirow{3}{*}{21,22} & $>0$ & $>0$ & 23 & $21 \bullet$ & 24 & 22 & \multirow{2}{*}{23,24} \\
& & $<0$ & 23 & 21 & $24 \circ$ & 22 & \\
\cline { 3 - 8 } & $<0$ & $>0$ & 24 & 22 & $23 \circ$ & 21 & \\
\cline { 3 - 8 } & & $<0$ & 24 & $22 \bullet$ & 23 & 21 & \\
\hline \multirow{2}{*}{23,24} & $>0$ & $>0$ & 22 & 24 & $21 \circ$ & 23 & \multirow{2}{*}{21,22} \\
\cline { 3 - 8 } & & $<0$ & 22 & $24 \bullet$ & 21 & 23 & \\
\cline { 3 - 8 } & \multirow{2}{*}{$<0$} & $>0$ & 21 & $23 \bullet$ & 22 & 24 & \\
\cline { 3 - 8 } & & $<0$ & 21 & 23 & $22 \circ$ & 24 & \\
\hline
\end{tabular}

\section{EXPERIMENTAL VERIFICATION}

The newly proposed PWM method and safe-commutation strategy were implemented on a $100 \mathrm{~W}$ HF-link converter prototype. Switching frequency was set to $f_{s 2}=200 \mathrm{kHz}$ on the secondary side with a ratio of $r=2$, leading to primaryside switching frequency of $f_{s 1}=100 \mathrm{kHz}$ synchronized with the triangular carrier of the secondary-side PWM modulator as described previously.

The switching pattern of the secondary-side bidirectional bridge, with $23 \& 24$ as outgoing switches and 21 \& 22 as incoming switches and positive voltage on the HF-link $v_{H F}>0$ is shown in Fig. 12. The waveforms can be compared with the switching sequence in Tab. I.

The reference voltage, output voltage, bridge voltage and HF-link voltage with zero reference voltage are given in Fig. 13.

The reference voltage and output voltage for a low voltage $400 \mathrm{~Hz}$ system is given in Fig. 14. 

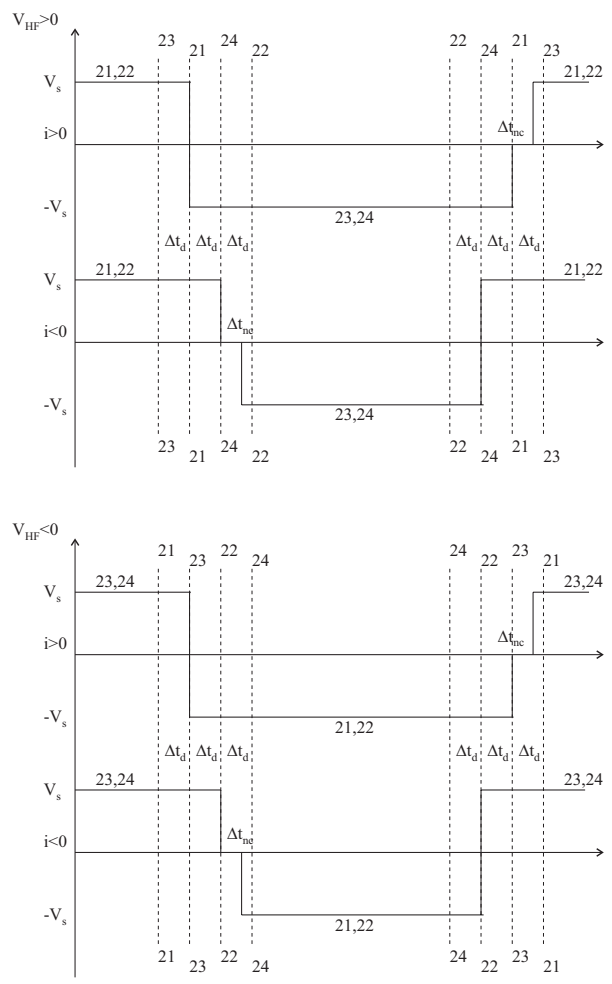

Fig. 11. Commutation diagram

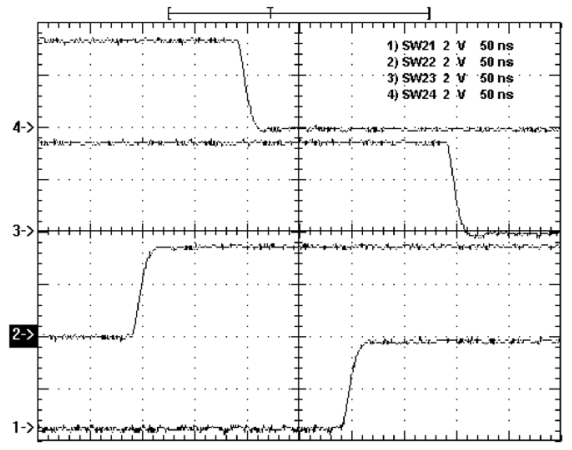

Fig. 12. Safe-commutation switching pattern

The presented measurements prove the feasibility of the proposed PWM method and safe commutation strategy for use in HF-link converters with non-modulated transformer voltages.

\section{CONCLUSION}

HF-link converter represents an interesting direct energy conversion topology, which is characterized by compactness, simplicity, high efficiency, high power density and low component count. It is therefore suitable for interfacing fuel cells and photovoltaics to different power systems without sacrificing their hardly achieved efficiency. This paper presented a new PWM method and safe-commutation strategy for HF-link converters with non-modulated transformer voltages. Their

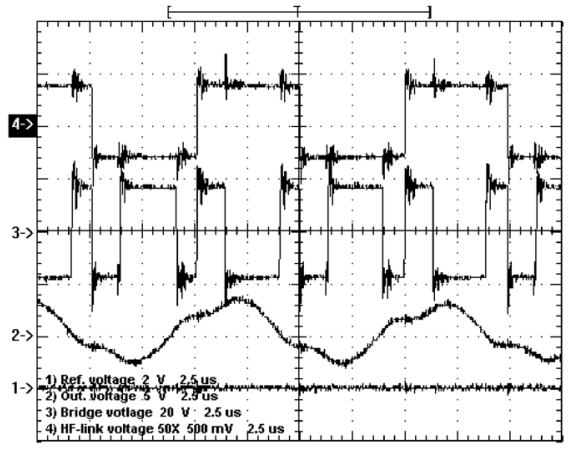

Fig. 13. Converter waveforms with zero reference voltage: 1) reference voltage, 2) output voltage 3 ) bridge voltage, 4) HF-link voltage

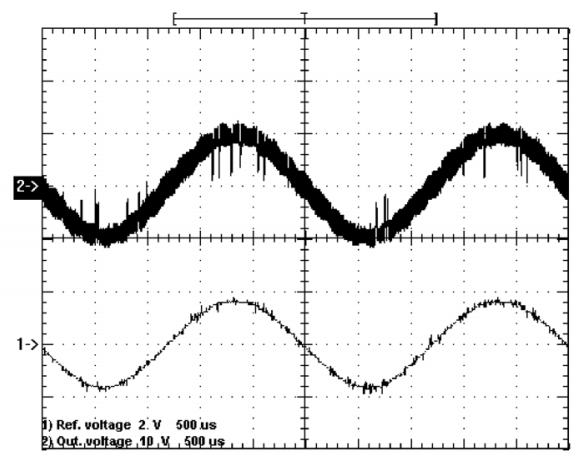

Fig. 14. Waveforms of the reference voltage (bottom) and output voltage (top) with $400 \mathrm{~Hz}$ reference

implementation is flexible and reliable, making it appropriate for many different HF-link converter topologies without relying on the selection of particular switching components. HF-link converter prototype with the proposed approach has been successfully realized.

\section{ACKNOWLEDGMENT}

The presented research in the field of HF-link conversion is part of the "SICAM - SIngle Conversion stage AMplifier" project at the Technical University of Denmark, funded under the grant of the Danish Energy Authority EFP no. 1273/020001 and performed in cooperation with Bang \& Olufsen ICEpower a/s in Kgs. Lyngby, Denmark.

\section{REFERENCES}

[1] P. Espelage and B. Bose, "High-frequency link power conversion," IEEE Transactions on Industry Applications, vol. IA-13, no. 5, pp. 387-94, 1977.

[2] D. Mitchell, "Dc to low frequency inverter with pulse width modulated high frequency link," U.S. patent 4,339,791, July 1982.

[3] T. Kawabata, K. Honjo, N. Sashida, K. Sanada, and M. Koyama, "High frequency link dc/ac converter with pwm cycloconverter," Industry Applications Society Annual Meeting, 1990., Conference Record of the 1990 IEEE, pp. 1119-1124 vol.2, 1990.

[4] M. Kneifel, M. Kokes, and D. Silber, "Half bridge for an integrable direct converter in high frequency link systems," Power Electronics Specialists Conference, 1996. PESC '96 Record., 27th Annual IEEE, vol. 1, pp. 462-468 vol.1, 1996. 
[5] I. Yamato, N. Tokunaga, Y. Matsuda, Y. Suzuki, and H. Amaro, "High frequency link dc-ac converter for ups with a new voltage clamper," Power Electronics Specialists Conference, 1990. PESC '90 Record., 21st Annual IEEE, pp. 749-756, 1990.

[6] T. Aoki, K. Yotsumoto, S. Muroyama, and Y. Kenmochi, "A new uninterruptible power supply with a bidirectional cycloconverter," Telecommunications Energy Conference, 1990. INTELEC '90., 12th International, pp. 424-429, 1990.

[7] B. Ozpineci and B. Bose, "Soft-switched performance-enhanced high frequency nonresonant link phase-controlled converter for ac motor drive," Industrial Electronics Society, 1998. IECON '98. Proceedings of the 24th Annual Conference of the IEEE, vol. 2, pp. 733-739 vol.2, 1998.

[8] P. Krein, R. Balog, and X. Geng, "High-frequency link inverter for fuel cells based on multiple-carrier pwm," Power Electronics, IEEE Transactions on, vol. 19, no. 5, pp. 1279-1288, 2004.
[9] P. Ljušev and M. Andersen, "Approaches to building single-stage ac/ac conversion switch-mode audio power amplifiers," in Proc. 11th International Power Electronics and Motion Control conference EPE-PEMC 2004, (Riga, Latvia), September 2-4 2004.

[10] P. Ljušev and M. Andersen, "Direct-conversion switching-mode audio power amplifier with active capacitive voltage clamp," in Proc. 36th IEEE Power Electronics Specialists Conference PESC 2005, (Recife, Brazil), June 12-16 2005.

[11] B. E. Attwood, L. E. Hand, and L. C. Santillano, "Audio amplifier with phase modulated pulse width modulation," U.S. patent 4,992,751, February 1991.

[12] P. Ljušev and M. Andersen, "Safe-commutation principle for direct single-phase ac-ac converters for use in audio power amplification," in Proc. Nordic Workshop on Power and Industrial Electronics NORPIE 2004, (Trondheim, Norway), June 14-16 2004. 